\title{
SPACE INVOLUTORIAL TRANSFORMATIONS OF THE GEISER AND BERTINI TYPES*
}

BY L. A. DYE

1. Introduction. One form of generalization of a plane involution is a space involutorial transformation in which each plane of a pencil is invariant and in each such plane there is a plane involution of the same type. Particular examples of this for Geiser and Bertini involutions have been given by Carroll, $\uparrow$ Snyder and Lehr, $\ddagger$ and Sharpe and Dye. $\S$ I shall discuss a more general form for space involutorial transformations arising from these plane involutions by means of a mapping on a cubic surface.ll The Bertini transformation obtained has the signature

$$
I_{120 n+51}: l^{120 n+34+6 t}+(O, \bar{O})^{120 n+40}+C_{12 n+6}^{6} ;
$$

the Geiser transformation has the signature

$$
I_{24 n+19}: l^{24 n+11+3 t}+O^{24 n+14}+C_{12 n+6}^{3} .
$$

2. The Geiser Transformation. In an involutorial space transformation of the Geiser type, let $x_{4}=\lambda x_{3}$ be the equation of the invariant pencil of planes, and let $I_{G}$ be the Geiser involution in the plane $x_{4}=\lambda x_{3}$. Choose one of the fundamental points of $I_{G}$ as $O \equiv(1, \lambda, 0,0)$ on the line $l \equiv x_{3}=x_{4}=0$, and map the $I_{G}$ on a cubic surface $F_{3}$ by means of the bilinear $T_{3-3}$ defined by the matrices

$$
\begin{array}{llll}
\|\left(a_{i 1} y_{i}\right) & \left(a_{i 2} y_{i}\right) & \left(a_{i 3} y_{i}\right) & \left(a_{i 4} y_{i}\right) \|, \\
\|\left(a_{1 i} x_{i}\right) & \left(a_{2 i} x_{i}\right) & \left(a_{3 i} x_{i}\right) & \left(a_{4 i} x_{i}\right) \|,
\end{array}
$$

* Presented to the Society, December 27, 1934.

$\dagger$ E. T. Carroll, American Journal of Mathematics, vol. 54 (1931), pp. 707717, and vol. 56 (1934), pp. 96-108.

¥ V. Snyder and M. Lehr, American Journal of Mathematics, vol. 53 (1931), pp. $186-195$.

$\S$ F. R. Sharpe and L. A. Dye, Transactions of this Society, vol. 36 (1934), pp. 292-305.

|| For a discussion of the mapping of a Geiser or a Bertini plane involution on a cubic surface, see H. F. Baker, Principles of Geometry, vol. 6, pp. 122-130. 
where here and in the rest of the paper only the first row of determinants and matrices is written; the second and third rows are obtained by replacing $a_{i j}$ by $b_{i j}$ and $c_{i j}$, respectively. To the plane $x_{4}=\lambda x_{3}$ corresponds the surface

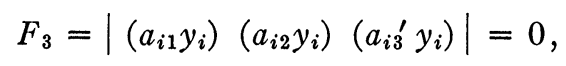

where $a_{i 3}^{\prime}=a_{i 3}+\lambda a_{i 4}$. The point $O$ goes into the point $P \equiv\left(D_{i}\right)$, where the $D_{i}$ are the third-order determinants of the matrix $\left\|\begin{array}{llll}a_{11}^{\prime} & a_{21}^{\prime} & a_{31}^{\prime} & a_{41}^{\prime}\end{array}\right\|$, and where $a_{i 1}^{\prime}=a_{i 1}+\lambda a_{i 2}$. The image of the line $l$ is the cubic curve $C_{3} \equiv\left\|\left(a_{i 1} y_{i}\right)\left(a_{i 2} y_{i}\right)\right\|=0$. The pairs of corresponding points of the Geiser involution $J_{G}$ on the surface $F_{3}$ are determined by the pairs of intersections with $F_{3}$ of lines through $P$.

In the $J_{G}$ on $F_{3}$ the image of $P$ is the cubic curve cut from $F_{3}$ by the tangent plane $p$ at $P$. The equation of $p$ is

$$
\mid \begin{array}{lll}
\left(a_{i 1}^{\prime} y_{i}\right) & \left(a_{i 2} D_{i}\right) & \left(a_{i 3}^{\prime} D_{i}\right) \mid=0 .
\end{array}
$$

The quadric cone $H$ through $C_{3}$ with vertex at $P$ has the equation

$$
\left|\left(a_{i 1}^{\prime} y_{i}\right)\left(a_{i 2} y_{i}\right)\left(a_{i 2} D_{i}\right)\right|=0,
$$

and meets $F_{3}$ in a residual cubic which is the image of $C_{3}$. The invariant curve of the $J_{G}$ is cut from $F_{3}$ by the polar quadric of $P$ with respect to $F_{3}$. This quadric $K$ has for its equation

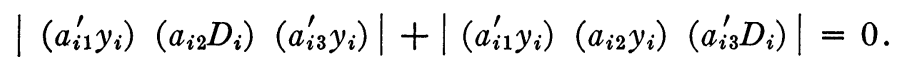

If $a_{i j}, b_{i j}$, and $c_{i j}$ are polynomials of order $n$ in $\lambda$, and if $\lambda$ is replaced by $x_{4} / x_{3}$, then the surfaces $y_{i}=0$ in the $(x)$ space are of order $3 n+3$. These surfaces have $l$ as a $3 n$-fold line and contain a curve $C_{12 n+6}$ of order $12 n+6$, of genus $24 n+3$, which meets $l$ in $12 n$ points. Since any plane through $l$ is invariant under the Geiser space transformation, there is a pencil of homaloidal surfaces consisting of the pencil of planes through $l$ and the images of $l$ and $O$. The equations of these image surfaces are obtained by replacing $y_{i}$ in the equations of $H$ and $p$ by the thirdorder determinants of the matrix (2). Since $H, p$, and $K$ are of orders $12 n+3,12 n+7$, and $12 n+4$ in $\lambda$, respectively, they correspond to surfaces of orders $12 n+8,12 n+10$, and $12 n+10$. 
The table of characteristics of the space involutorial transformation $I_{24 n+19}$ can now be written:

$$
\begin{aligned}
& O \sim F_{12 n+10}: l^{12 n+7+2 t}+O^{12 n+9}+C_{12 n+6}, \\
& l \sim F_{12 n+8}: l^{12 n+3+t}+O^{12 n+4}+C_{12 n+6}^{2}, \\
& C_{12 n+6} \sim F_{60 n+44}: l^{60 n+26+6 t}+O^{60 n+32}+C_{12 n+6}^{7}, \\
& S_{1} \sim S_{24 n+19}: l^{24 n+11+3 t}+O^{24 n+14}+C_{12 n+6}^{3}, \\
& K_{12 n+10}: l^{12 n+4+2 t}+O^{12 n+6}+C_{12 n+6}^{2},
\end{aligned}
$$

where the coefficient of $t$ indicates the number of fixed tangent planes at a point of $l$. The image of the $C_{12 n+6}$ is obtained by applying the transformation to an $S_{24 n+19}$.

The parasitic lines of the transformation consist of the trisecants of $C_{12 n+6}$ which meet $l$, and the bisecants of $C_{12 n+6}$ which pass through $O$. The number of trisecants of a $C_{m}$ which meet a line $l$ having $i$ intersections with $C_{m}$ is*

$(m-2)[h-m(m-1) / 6]-i(h-m+2)+i(i-1)(i-2) / 6$,

where $h$ is the number of apparent double points of $C_{m}$. The number of trisecants of $C_{12 n+6}$ which meet $l$ is $24 n+8$. In order to obtain the number of bisecants through $O$, it is necessary to set up a correspondence. Given a point $\mu$ on the line $l$, there are $h^{\prime}=h-12 n(12 n-1) / 2=36 n+7$ bisecants through it which determine $h^{\prime}$ planes $\lambda$. Given a plane $\lambda$ through $l$ there are six intersections of $C_{12 n+6}$ with it not on $l$ and hence fifteen bisecants which determine fifteen points $\mu$. In the $(\lambda, \mu)$ correspondence there are $h^{\prime}+15=36 n+22$ coincidences. There are then $36 n+22$ bisecants of $C_{12 n+6}$ which meet $O$, and the total number of parasitic lines is $60 n+30$.

Let $\zeta$ be the number of parasitic conics of the transformation, and let $\eta$ be the number of parasitic cubics. The complete intersection of two surfaces of the web of $S_{24 n+19}$ is made up of

$$
\begin{gathered}
(24 n+19)^{2}=24 n+19+(24 n+11)^{2}+9+9(12 n+6) \\
+60 n+30+8 \zeta+27 \eta
\end{gathered}
$$

* L. A. Dye, this Bulletin, vol. 41 (1935), pp. 109-110. 
curves, and the complete intersection of an $S_{24 n+19}$ and the $K_{12 n+10}$ is made up of

$$
\begin{array}{r}
(24 n+19)(12 n+10)=12 n+10+(12 n+4)(24 n+11) \\
+6+6(12 n+6)+60 n+30+4 \zeta+9 \eta
\end{array}
$$

curves. The solution of these equations is $\zeta=24 n+16, \eta=0$; therefore there are $24 n+16$ conics and $60 n+30$ lines of the second species in the $I_{24 n+19}$.

3. The Bertini Transformation. The methods of the last section are now used to study an involutorial space transformation of the Bertini type. Let the invariant pencil of planes have the line $l \equiv x_{3}=x_{4}=0$ as axis. In a plane $x_{4}=\lambda x_{3}$ let $O \equiv(1, \mu, 0,0)$ and $\bar{O} \equiv(1,-\mu, 0,0),\left(\mu^{2}=\lambda\right)$, be two fundamental points of the Bertini involution $I_{B}$ in the plane. The $I_{B}$ is mapped on the cubic surface (3) by means of the $T_{3-3}$ defined by the matrices (1) and (2). The images of $O$ and $\bar{O}$ are $P \equiv\left(D_{i}\right)$ and $\bar{P} \equiv\left(\bar{D}_{i}\right)$; $D_{i}$ and $\bar{D}_{i}$ are the third-order determinants of the matrices

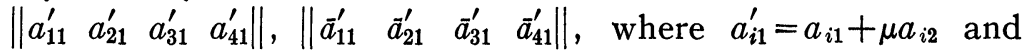
$\bar{a}_{i 1}=a_{i 1}-\mu a_{i 2}$. The image of $l$ is the $C_{3}$ given by the matrix equation $\left\|\left(a_{i 1} y_{i}\right)\left(a_{i 2} y_{i}\right)\right\|=0$.

The tangent planes to $F_{3}$ at $P, \bar{P}$ are $p, \bar{p}$ and they have as equations

$$
\begin{aligned}
& p \equiv\left|\left(a_{i 1}^{\prime} y_{i}\right)\left(\bar{a}_{i 1}^{\prime} D_{i}\right)\left(a_{i 3}^{\prime} D_{i}\right)\right|=0, \\
& \bar{p} \equiv\left|\left(a_{i 1}^{\prime} \bar{D}_{i}\right)\left(\bar{a}_{i 1}^{\prime} y_{i}\right)\left(a_{i 3}^{\prime} \bar{D}_{i}\right)\right|=0 .
\end{aligned}
$$

We now define two numbers $d, \bar{d}$ as follows:

$$
\begin{aligned}
& d \equiv p\left(\bar{D}_{i}\right) \equiv\left|\left(a_{i 1}^{\prime} \bar{D}_{i}\right)\left(\bar{a}_{i 1}^{\prime} D_{i}\right)\left(a_{i 3}^{\prime} D_{i}\right)\right|, \\
& \bar{d} \equiv \bar{p}\left(D_{i}\right) \equiv\left|\left(a_{i 1}^{\prime} \bar{D}_{i}\right)\left(\bar{a}_{i 1}^{\prime} D_{i}\right)\left(a_{i 3}^{\prime} \bar{D}_{i}\right)\right| .
\end{aligned}
$$

The residual intersection $R$ of the line $P \bar{P}$ with $F_{3}$ has as coordinates $\left(\bar{d} D_{i}-d \bar{D}_{i}\right)$. The equation of the tangent plane to $F_{3}$ at $R$ is

$$
\begin{aligned}
& r \equiv d^{2} \bar{p}+\bar{d}^{2} p-d \bar{d}\left\{\left|\left(a_{i 1}^{\prime} y_{i}\right)\left(\bar{a}_{i 1}^{\prime} D_{i}\right)\left(a_{i 3}^{\prime} \bar{D}_{i}\right)\right|\right. \\
&\left.+\left|\left(a_{i 1}^{\prime} \overline{D_{i}}\right)\left(\bar{a}_{i 1}^{\prime} y_{i}\right)\left(a_{i 3}^{\prime} D_{i}\right)\right|+\left|\left(a_{i 1}^{\prime} \bar{D}_{i}\right)\left(a_{i 1}^{\prime} D_{i}\right)\left(a_{i 3}^{\prime} y_{i}\right)\right|\right\}=0 .
\end{aligned}
$$

The pairs of corresponding points in the Bertini involution $J_{B}$ on $F_{3}$ are cut out by the conics tangent to $F_{3}$ at $P$ and $\bar{P}$. 
The image of $P$ in $J_{B}$ is the sextic cut from $F_{3}$ by the quadric having contact with $F_{3}$ at $\bar{P}$ and contact of the second order at $P$. Its equation is

$$
\begin{aligned}
H \equiv \bar{d}^{2} d & {\left[\left|\left(a_{i 1}^{\prime} y_{i}\right)\left(\bar{a}_{i 1}^{\prime} D_{i}\right)\left(a_{i 3}^{\prime} y_{i}\right)\right|\right.} \\
& \left.+\left|\left(a_{i 1}^{\prime} y_{i}\right)\left(\bar{a}_{i 1}^{\prime} y_{i}\right)\left(a_{i 3}^{\prime} D_{i}\right)\right|\right]+p r=0 ;
\end{aligned}
$$

similarly the quadric which determines the image of $\bar{P}$ has the equation

$$
\begin{aligned}
\bar{H} \equiv d^{2} \bar{d}\left[\left|\begin{array}{lll}
\left.\mid a_{i 1}^{\prime} \bar{D}_{i}\right) & \left(\bar{a}_{i 1}^{\prime} y_{i}\right) & \left(a_{i 3}^{\prime} y_{i}\right)
\end{array}\right|\right. \\
+\mid \begin{array}{lll}
\left(a_{i 1} y_{i}\right) & \left(\bar{a}_{i 1}^{\prime} y_{i}\right) & \left.\left(a_{i 3}^{\prime} \bar{D}_{i}\right) \mid\right]+p r=0 .
\end{array}
\end{aligned}
$$

The cubic curve $C_{3}$ corresponds to a cubic cut from $F_{3}$ by the quadric through $C_{3}$ and touching $F_{3}$ at $P$ and $\bar{P}$. This quadric has the equation

$$
L \equiv\left|\left(a_{i 1}^{\prime} y_{i}\right)\left(a_{i 1}^{\prime} y_{i}\right) A\right|=0
$$

where $A, B, C$ are the second-order determinants of a two column matrix whose columns are made up of the second-order determinants of the matrix $\left\|\left(\bar{a}_{i 1}^{\prime} D_{i}\right) \quad\left(a_{i 3}^{\prime} D_{i}\right)\right\|$ and the matrix $\left\|\left(a_{i 1}^{\prime} \bar{D}_{i}\right) \quad\left(a_{i 3}^{\prime} \bar{D}_{i}\right)\right\|$. The web of quadrics which touch $F_{3}$ at $P$ and $\vec{P}$ cuts $F_{3}$ in a web of sextic curves of genus two which is invariant under $J_{B}$. The locus of an additional point of contact with $F_{3}$ of quadrics of the web is the invariant nonic of $J_{B}$. It lies on the cubic surface $K$ which has the equation

$$
p \bar{H}-\bar{p} H=0 \text {. }
$$

If the $a_{i j}, b_{i j}$, and $c_{i j}$ are polynomials of order $n$ in $\lambda$, the surfaces $H, \bar{H}, L$, and $K$ are of orders $48 n+13,48 n+13,24 n+6$, and $36 n+9$ in $\lambda$. When $\lambda$ is replaced by $x_{4} / x_{3}$, then as in $\$ 2$ we can write the table of characteristics of the involutorial space transformation $I_{120 n+51}$ as follows:

$$
\begin{aligned}
& l \sim F_{24 n+12}: l^{24 n+7+t}+(O, \bar{O})^{24 n+8}+C_{12 n+6}^{2} \\
&(O, \bar{O}) \sim F_{96 n+38}: l^{96 n+26+5 t}+(O, \bar{O})^{96 n+31}+C_{12 n+6}^{4}, \\
& C_{12 n+6} \sim F_{264 n+112}: l^{264 n+76+12 t}+(O, \bar{O})^{264 n+88}+C_{12 n+6}^{13} \\
& S_{1} \sim S_{120 n+51}: l^{120 n+34+6 t}+(O, \bar{O})^{120 n+40}+C_{12 n+6}^{6}, \\
& K_{36 n+18}: l^{36 n+9+3 t}+(O, \bar{O})^{36 n+12}+C_{12 n+6}^{3} .
\end{aligned}
$$


The parasitic lines of the transformation are of three types. (a) There are the $24 n+8$ trisecants of the $C_{12 n+6}$. (b) The bisecants of $C_{12 n+6}$ through $O$ or $\bar{O}$ are parasitic. In a plane $\lambda$ through $l$ there are fifteen bisecants through the six points of $C_{12 n+6}$ not on $l$, which determine fifteen points $\mu$ on $l$. Given a point $\mu$ on $l$ there are $h^{\prime}=36 n+7$ bisecants of $C_{12 n+6}$ through it which determine $h^{\prime}$ planes $\lambda$. In the $(\lambda, \mu)$ correspondence there are $15+2 h^{\prime},\left(2 h^{\prime}\right.$, since $\left.\mu^{2}=\lambda\right)$, coincidences, or $72 n+29$ positions of the points $O, \bar{O}$ such that bisecants of $C_{12 n+6}$ may be drawn through them. (c) In each of the $12 n$ planes determined by $l$ and the tangents to $C_{12 n+6}$ at its $12 n$ intersections with $l$, the Bertini involution in the plane breaks down, and the line $l$ is shed off. Hence there are $12 n$ parasitic lines consecutive to $l$ in these $12 n$ planes. The total number of parasitic lines is $108 n+37$.

To determine the number of parasitic conics and cubics we take the complete intersection of two $S_{120 n+51}$ and an $S_{120 n+51}$ with $K_{36 n+18}$.

$$
\begin{aligned}
(120 n+51)^{2}=120 n+51+(120 n+34)^{2}+36+36(12 n+6) \\
+108 n+73+8 \zeta+27 \eta \\
(120 n+51)(36 n+18)=36 n+18+(36 n+9)(120 n+34) \\
+18+18(12 n+6)+108 n+37+4 \zeta+9 \eta .
\end{aligned}
$$

The solution of these equations is $\zeta=144 n+47$ conics and $\eta=84 n+27$ cubics. The fundamental curves of the second species of the involutorial transformation $I_{120 n+51}$ consist of $108 n+37$ lines, $144 n+47$ conics, and $84 n+27$ cubics.

Cornell University 